\title{
Mechanistic Classification of Cement Treated Base in
}

\section{Western Australia}

\author{
Yang Sheng Yeo, P. Jitsangiam and H. Nikraz \\ Department of Civil Engineering, Curtin University of Technology, Australia
}

\begin{abstract}
In the past decade alone, the BITRE has indicated an increase of $40 \%$ in road users, escalating demands for quality pavements to service unprecedented traffic conditions. An abundance of crushed rocks are available in Western Australia but do not meet strength requirements for road construction. However, cement treatment of crushed rocks, forming Cement Treated Crushed Rocks (CTCR), improves the mechanical properties of the material, allowing wider application. In order to streamline the mix design of CTCR, the classification of its behaviour is pivotal. Austroad classifies cement treated pavement materials as either being modified or bound based on its Unconfined Compressive Strength (UCS) and performance attributes. Bound materials are defined by its susceptibility to fatigue failure which, in the mechanistic-empirical design for flexible pavements, is dictated by the flexural modulus. However, in the study of damage mechanics, fatigue life is suggested to be an accumulation of micro-scale damage in lieu of dependency to ultimate stresses. Strain dependent damage functions are used phenomologically to explain the evolution of fatigue for various engineering materials. This paper therefore investigates a theoretical relationship between strain and fatigue life prediction supported by a laboratory investigation on the use of UCS for classification. This is achieved by providing regression analysis with strain parameters used in fatigue life prediction. The Indirect Tensile Strength (ITS) test is also employed to this end. It is observed that strain at onset of micro-cracking coalescence $\left(\varepsilon_{30}\right)$ is independent of test type undertaken and potentially capable of acting as a more superior blanket classification for cemented materials.
\end{abstract}

Key words: Cement treated materials, unconfined compressive strength, fatigue

\section{Introduction}

In the past decade alone, the BITRE [1] has indicated an increase of $40 \%$ in road users, escalating demands for quality pavements to service unprecedented traffic conditions. Western Australia has an abundance of crushed rocks but nevertheless do not necessarily meet performance requirements for heavy traffic loads. When cement is treated to crushed rocks an interlocking matrix between the aggregates is created, forming Cement Treated Crushed Rocks (CTCR). This allows the otherwise mechanically unsuitable material to be used in road construction.

Nonetheless, Main Roads Western Australia (MRWA) limits the use of CTCR due to its tendency to undergo shrinkage and fatigue failure. With the lack of

Corresponding author: Yang Sheng Yeo, BEng, PhD, research fields: geotechnical, pavements. E-mail: yangsheng.yeo@postgrad.curtin.edu.au. interest, very limited studies of CTCR exist in Western Australia. This paper presents a theoretical development and laboratory investigation to examine the applicability of the existing Austroads recommended classifications of cement stabilisation in WA.

\section{Classification of Cement Treated Pavement Materials}

Pavement materials are traditionally recognised as either exhibiting unbound or bound behaviours. It is this alteration of mechanical behaviour that forms the basis of CTCR classification. The degree of binding is designated as either being "modified" or "stabilised".

Modification is a result of applying small amounts of cementitious binders, typically lime or chemical binders [2, 3]. It is not intended to improve mechanical (strength) performance, but instead aims to reduce 
plasticity and moisture susceptibility [2], thus minimising rutting potential and increasing durability.

Stabilisation is the addition of cementitious binders so that improvements in mechanical performance (strength) are achieved. The level of stabilisation is also differentiated to two broad categories, i.e., lightly stabilised and stabilised [2].

In pavement mix design, the distinction of the mechanical behaviour under varying cement content is particularly important to the industry as it dictates the cost, serviceability and design life of a road. However, there is no exact distinguishing factor between each category because of overlapping traits [3].

\section{Austroads Classification Criteria}

The identification of classification points has generally been empirical in nature and primarily based on experience. Austroads Pavement Design Guideline classifies cemented materials based on its unconfined compressive strength (UCS) and performance attributes. The recent classification guideline, adapted from Austroads [2], is presented in Table 1 below.

UCS is widely accepted as the classification criterion for cemented materials within the transportation industry because of its relative ease and speed to undertake [4]. The standardisation of procedures (AS5101.4) and the availability of testing frames or moulding equipment in typical geotechnical laboratories are added advantages. Nonetheless, variations of sample preparation methods and limiting UCS ranges exist across the different states in Australia and internationally [4]. Although they are in general agreement of the Austroads recommendations, the

Table 1 Austroad [2] classification of cemented materials.

\begin{tabular}{|l|l|l|}
\hline Classification & Testing Criteria & Performance Attributes \\
\hline Modified $^{1}$ & $0.7 \mathrm{MPa}<\mathrm{UCS}^{2}<1.5 \mathrm{MPa}$ & $\begin{array}{l}\text { Flexible pavement subject to shear failure within pavement layers } \\
\text { and/or subgrade deformation. }\end{array}$ \\
\hline $\begin{array}{l}\text { Lightly Bound } \\
\text { Stabilised) }\end{array}$ & $1.5 \mathrm{MPa}<\mathrm{UCS}^{2}<3 \mathrm{MPa}$ & $\begin{array}{l}\text { Lightly bound pavement which may be subject to tensile fatigue or } \\
\text { subgrade deformation. }\end{array}$ \\
\hline Bound (Stabilised) & $\mathrm{UCS}^{2}>3 \mathrm{MPa}$ & $\begin{array}{l}\text { Bound pavement which may be subject to tensile fatigue cracking and } \\
\text { transverse dry shrinkage cracking. }\end{array}$ \\
\hline
\end{tabular}

${ }^{1}$ Modification is typically achieved by addition of lime, polymer or chemical binders.

${ }^{2}$ Values determined from test specimens stabilised with GP cement and prepared using Standard compactive effort, normal curing for a minimum 28 days and 4 hour soak conditioning. variability of the parameters are a clear reflection on the uncertainty in defining CTCR behaviour.

The Unconfined Compressive Strength (UCS) provides an indicative measure of the normal stress and cohesive shear strength of the cement matrix, which has been believed to express the degree of binding achieved. It does not provide any input to design, but is primarily used for classification.

In retrospect to its predecessors, the current classification provides a performance description of class, which highlights the failure mechanism of the material. It supersedes the previous approach which was based on UCS and design flexural modulus. The flexural modulus has since been removed as a classifying criterion and instead reflected as a parameter in calculating the fatigue life of bound materials shown below.

$$
N=R F\left[\frac{1130_{0} / E^{0.80_{4}}+191}{\mu \varepsilon}\right]^{12}
$$

Where, $E=$ flexural modulus

$\mu \varepsilon=$ load-induced strain

$\mathrm{RF}=$ reliability factor for cemented materials fatigue

This paper is in agreement with the performance attributes as a classification methodology as seen in Table 1, in particular the postulate that tensile fatigue failure is the defining criterion for the identification of stabilisation. Nevertheless, the validity of the testing criteria is investigated. A phenomological theory development and preliminary laboratory investigation is undertaken. 


\section{Classical Mechanical Parameters vs. Damage Evolution}

Austroad mechanistic design guidelines idealises the structural analysis of pavements using a multi-layered model [2] as shown in Fig. 1. Within the model, cemented base courses are characterised as bound materials having developed tensile strength from the formation of interlocking cement matrices between aggregates. The critical response of this layer is the tensile strains at the base of the layer, where distress is manifested as a bottom-up fracture. The model further assumes the pavement layer to be homogeneous, elastic and isotropic.
In distinct contrast to the idealised model, the fatigue mechanism of stabilised materials has been better characterised as a reduction in stiffness caused by an accumulation of damage in lieu of distinct transverse ruptures normally seen from ultimate loadings as discussed in the following subsection. The accumulation of damage by the pavement structure from repeated loads ultimately reaches a distress limit whereby the cement matrix disintegrates, consequently resulting in aggregates returning to its original unbound mechanical state, a service stage known as equivalent granular phase.

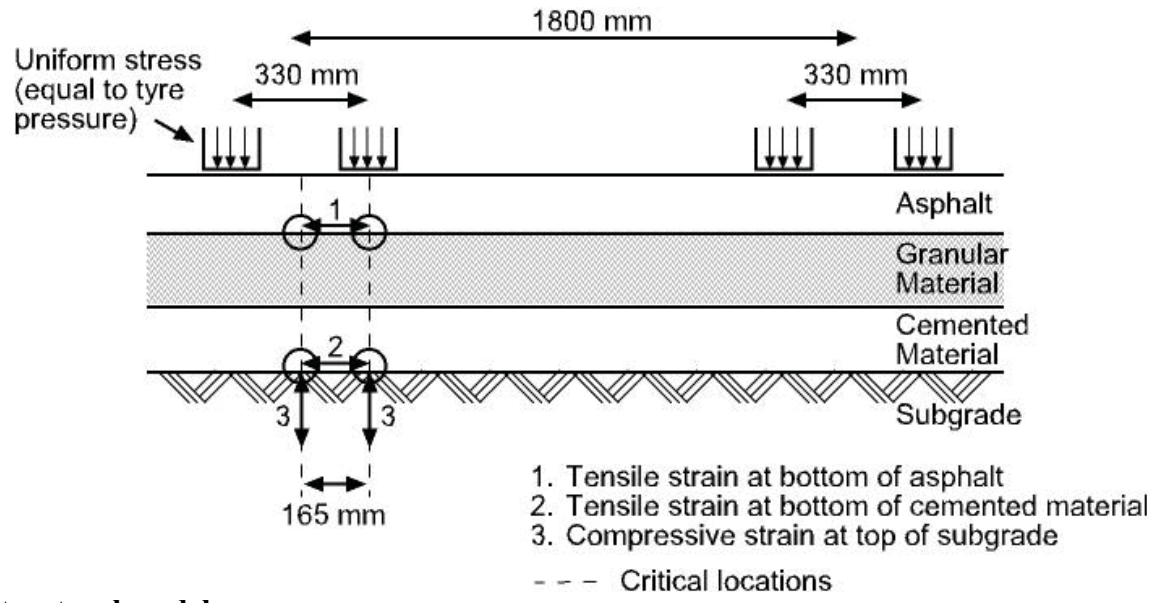

Fig. 1 Pavement structural model.
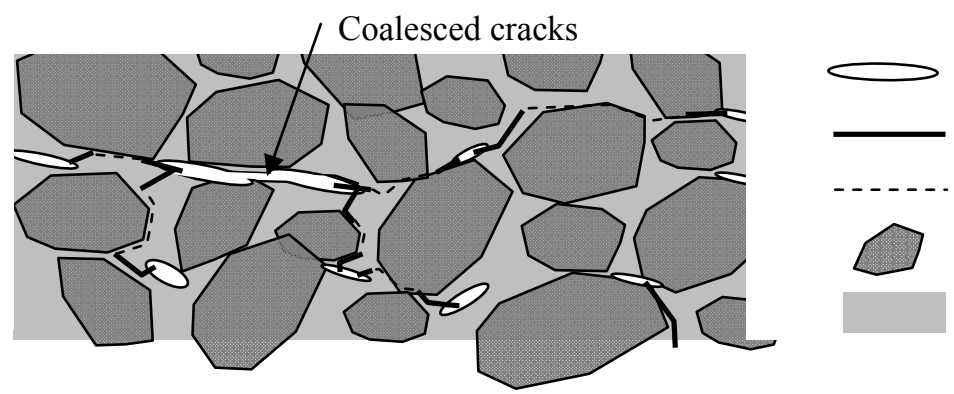

Existing microcracks, $\mathrm{E}=0$

Fracture process zone, $\mathrm{E}=\mathrm{E}$

Undamaged crack path, $\mathrm{E}=\mathrm{E}_{0}$

Crushed rock aggregates

Cement matrix

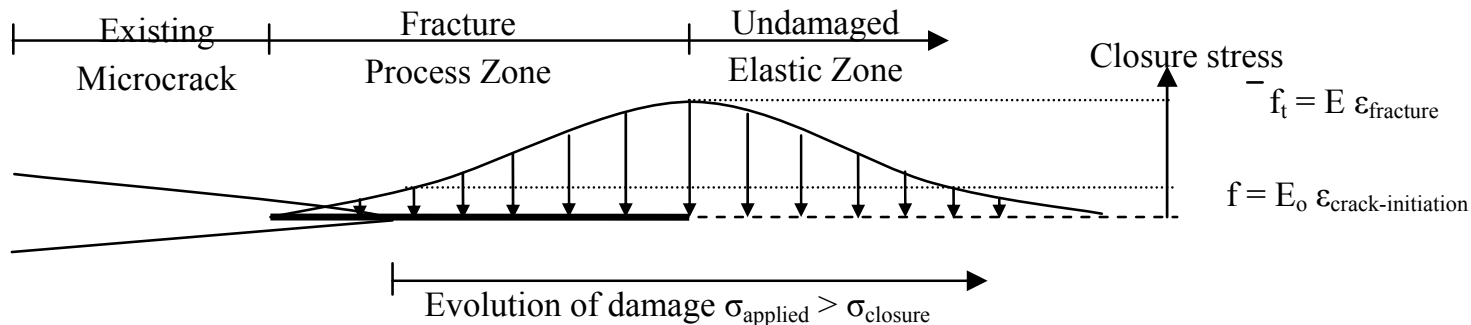

Fig. 2 Micro-cracking fictitious crack model. 


\section{Fracture and Damage Mechanics for Cement Treated Crushed Rocks}

In the study of continuum damage mechanics, fatigue is characterised by the accumulation of damage on a micro-scale. The quasi-brittle nature of CTCR causes the material to undergo damage in the form of nucleation of voids formed from the coalescence of microcracks [5]. This fracture process involves creation of new surfaces in the material, a phenomenon much better described by energy principles than by classical mechanics [6, 7].

Moreover, based on the strain equivalence principle $[7,8]$, the concept of damage can be represented by introducing a damage function. It is derived on the basis that the virgin material and its continuum model must contain equal strain energies when subjected to similar global displacements. It is represented based on the degradation of elastic modulus which results in a lowered capacity to store strain energy, i.e.,

$$
\bar{E}=E_{0}(1-D)
$$

The strain behaviour of a damaged material may therefore be represented as follow,

$$
\varepsilon=\frac{\sigma}{\bar{E}}=\frac{\sigma}{E_{0}(1-D)}=\frac{\bar{\sigma}}{E}
$$

As shown in equation above, prior to the onset of damage, i.e., $\mathrm{D}=\mathrm{D}_{\mathrm{o}}=0$, the linear elastic postulate is observed. This damage limit is the endurance limit of CTCR basecourse. To understand the endurance limit or the onset of damage, the fictitious model developed by [9] is referred. A model of the damage initiation is shown in the Fig. 2 adapted from Gdoutos [10].

The development of cracking on a micro-scale is as shown in Fig. 2. The endurance limit is exceeded when the applied stresses exceed the closure stress at the tip of the existing micro-crack, thus inflicting permanent deformation to the cement matrix. Similarly for concrete, the above model is used to predict the endurance limit or tensile capacity of concrete, estimated at $0.33 f_{t}$. Nevertheless, an inherent relationship exists between CTCR and fatigue. This is further discussed in the next section.

\section{The Fatigue and Strain Relationship}

The phenomology of fatigue generally observes a power law such as first noted in classic fracture mechanics by the Paris Law. However, as described in the preceding section, effective stress of damaged quasi-brittle materials can be represented by strain. This is supported by successfully characterisation fatigue life of cemented materials based on a logarithmic function of strain, to name a few Kaplan [11], Austroads [2], Alliche and François [6], and AARB [12].

More significantly, the strain measured at the onset of damage denoted by the termination of elastic linearity (or the endurance limit as defined earlier) is observed as a material constant [11-13]. The methodology adopted by Kaplan [11] measures the cease in proportionality of the stress strain curve. This requires sound judgement and precise measurement of strain, a difficult procedure to repeat. On the other hand, [14] shown that the proportionality of stress strain relationships ceases generally at $30 \%$ of the maximum load, a simplified assumption that is adopted by this paper which also conforms to the $0.33 f_{t}$ estimated for concrete tensile cracking.

This is in contrast to ultimate stresses and strain which are dependent of the type of test undertaken [13]. Given that the classification of CTCR is dependent on ultimate stresses, it is thus the interest of this paper to investigate the compatibility of UCS to material classification and investigate instead a plausible categorisation using strain of the endurance limit denoted as the initiation of damage.

\section{Laboratory Regime}

Given the strong dependence of fatigue life prediction to strain, an investigation between the relationship of the existing classification criteria and strain is undertaken. A regression analysis is investigated between unconfined compressive strength (UCS) and indirect tensile strength (ITS) against strain at the crack initiation or onset of damage, defined as the 
strain at $30 \%$ of maximum load $\left(\varepsilon_{30}\right)$, and strain at failure, measured at $95 \%$ of maximum load $\left(\varepsilon_{95}\right)$.

Two standardised tests are undertaken, i.e. the Unconfined Compressive Strength Test to AS5101.4 [15] and Indirect Tensile Strength Test to AS1012.10 [16]. Specimens are prepared as a function of cement content, ranging from $2 \%$, to $5 \%$ in $1 \%$ increments, compacted to $100 \%$ modified MDD, and cured 28 days prior to testing. Specimens are cured in a constant humidity environment and soaked 4 hours prior to testing. A minimum of 3 specimens are prepared for each mix design.

\section{Discussions Laboratory Results}

UCS and ITS results against cement content, $\varepsilon_{30}$ against cement content and $\varepsilon_{95}$ against cement content are plotted in Fig. 3.

Chart A indicates a statistically strong linear relationship between cement content and the two sets of stresses. Chart B shows a strong linear regression relationship between $\varepsilon_{30}$ and $\%$ cement with both tests showing very similar strain values. In contrast, strain near failure as shown in Chart $\mathrm{C}$, defined as strain under $95 \%$ of maximum loading $\left(\varepsilon_{95}\right)$, exhibits a statistically weak relationship to $\%$ cement.

Based on the recommendation by Austroads (refer Table 1), it is implied from the lab results that Western Australian CTCR are "heavily bound" even at very low cement content. This categorisation is inconsistent with the physical state of the material during the laboratory testing which exhibited very weak cohesion between aggregates and the matrix. The UCS values on a linear regression also do not intersect with the origin. These observations points to other latent factors contributing to the strength of the material, e.g., the compressive stresses being transferred through the aggregates and cement paste during UCS tests. It does not relate to the damage theory developed within this paper that fatigue life is a function of micro-crack coalescence occurring in the interfacial transition zone phase.
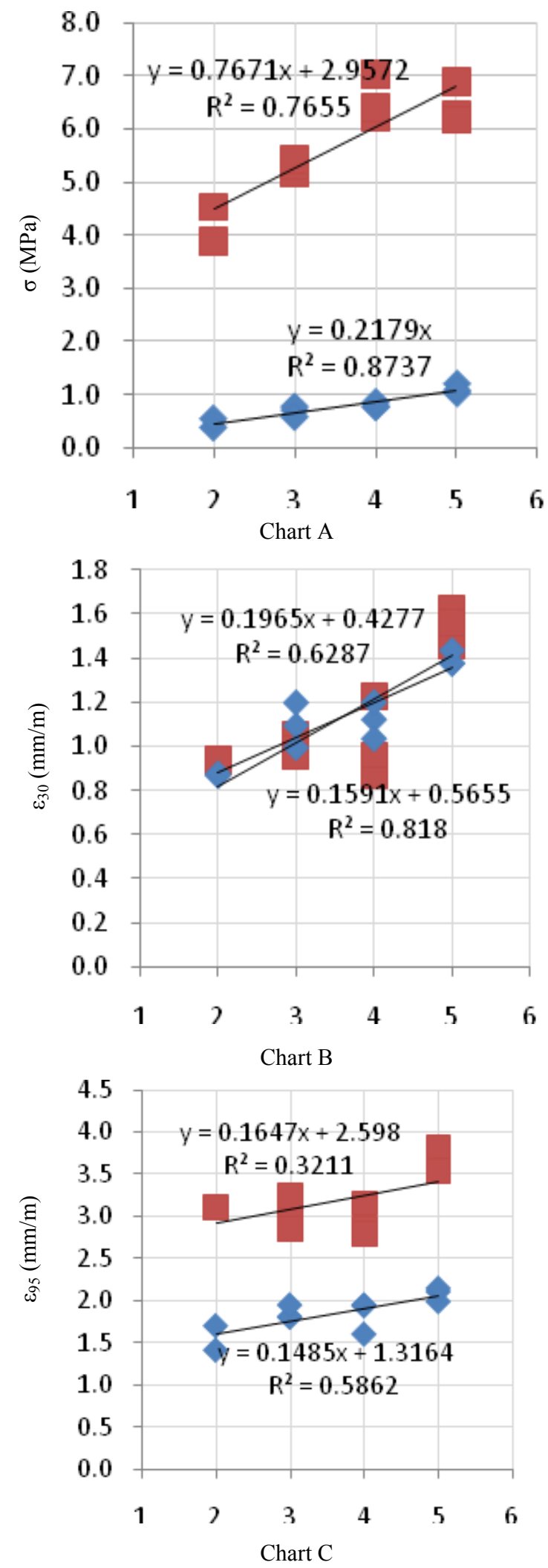

Fig. 3 Chart A: Stress vs. \% cement; Chart B: $\varepsilon_{30}$ vs. \% cement; Chart C: $\varepsilon_{95}$ vs. \% cement. 
More importantly, the laboratory investigation has showed that strain at the onset of cracking is a material constant, independent of type of test undertaken. This suggests that within the endurance limit, i.e. prior to damage, the cemented material acts homogeneously. This is in contrast to the strain at failure where a consistent gap is noted between the laboratory results.

\section{Recommendation and Conclusion}

The current classification of CTCR by ultimate stresses is suggested to be incompatible with principles of fracture and damage mechanics, which forms the postulate of fatigue life prediction. Instead, it is proposed that the strain at the endurance limit, i.e., prior to the onset of fatigue damage, typically measured at $30 \%$ of maximum load (denoted as $\boldsymbol{\varepsilon}_{\mathbf{3 0}}$ ) potentially be used as a blanket classification criteria for CTCR. It exhibits a statistically strong relationship with stresses and mix design (\% cement content) and acts independently from the type of test undertaken.

Based on the two tests undertaken, i.e., the Unconfined Compressive Strength (UCS) Test and Indirect Tensile Strength (ITS) Test, the strain at crack initiation for Western Australian CTCR, can be determined from the equation:

$$
\varepsilon_{30}=0.16 \times \% \text { cement content }+0565
$$

Since, it is well accepted and recognised that UCS is an economical and speedy testing regime, a further advantage to the proposed approach is that a current practices can be retained. The difference being a different value is taken from the investigation.

This paper has presented a simplified phenomological assertion of the relationship between strain and fatigue life based on fracture and damage mechanics. It is established that strain prior to the onset of failure is independent of type of test undertaken. As strain is an input into fatigue life prediction, its application as a classification criterion would also further benefit the design process.

Further testing is required to establish a suitable $\varepsilon_{30}$ range based on a fatigue damage model for CTCR, which will allow a more concise characterisation of the material. Further testing of other materials is also required to improve the validity of the proposed relationship.

\section{References}

[1] Bureau of Infrastructure, Transport and Regional Economics, Australian Transport Statistics Yearbook 2009, BITRE, Canberra, ACT.

[2] Austroads, Part 4D: stabilised materials. Guide to Pavement Technology, Austroads, Sydney NSW, 2006.

[3] K. Jenkins, Hitchiker's Guide to Pavement Engineering, Stellenbosch University, Matieland, 2002.

[4] G. Vorobieff, Mix design for stabilised pavement materials, Austroads AP-T16, 2002.

[5] T. J. Balbo and J. P. Cintra, Fatigue verification criteria for semi-rigid pavements, National Meeting on Asphalt Mixture and Pavements, available online at: http://www.ptr.usp.br/.

[6] A. Alliche and D. François, Damage on concrete in fatigue, Journal of Engineering Mechanics 118 (11) (1992) 2176-2190.

[7] U. Lee, G. A. Lesieutre and L. Fang, Anisotropic damage mechanics based on strain energy equivalence and equivalent elliptical microcracks, Interntional Journal of Solids Structures 34 (33-34) (1997) 4377-4397.

[8] F. Sirdoff, Description of anisotropic damage application to elasticity, Colloquim on Physical Nonlinearities in Structural Analysis, 1981, pp. 237-244.

[9] Z. Bazant, Concrete fracture models: testing and practice, Engineering Fracture Mechanics 69 (2002) 165-206.

[10] E. E. Gdoutos, Fracture Mechanics: An Introduction, Springer, Netherlands, 2005.

[11] M. F. Kaplan, Strain and stresses of concrete at initiation of cracking and near failure, Journal of American Concrete Institute 60 (1963) 853-880.

[12] ARRB Group Pty Ltd., Cost-effective structural treatments for rural highways: cemented materials interim report. Austroads, Sydney, NSW, 2010.

[13] B. L. Karihaloo and D. Fu, An anisotropic damage model for plain concrete, Engineering Fracture Mechanics 35 $(1 / 2 / 3)(1990)$ 205-209.

[14] F. Buktus, Reid highway basecourse test sections, Pavement Engineering Report No. 2004/17.

[15] Australian Standard, Method 4: unconfined compressive strength of compacted materials, Methods for preparation and testing of stabilized materials, AS 5101.4-2008.

[16] Australian Standard, Method 10: Determination of indirect tensile strength of concrete cylinders ("Brazil" or splitting test), Methods for testing concrete, AS 1012.10 2000. 